\title{
MINIMUM ENERGY CONTROL OF FRACTIONAL DESCRIPTOR POSITIVE DISCRETE-TIME LINEAR SYSTEMS
}

\author{
TADEUSZ KACZOREK \\ Faculty of Electrical Engineering \\ Białystok University of Technology, ul. Wiejska 45D, 15-351 Białystok, Poland \\ e-mail:kaczorek@isep.pw.edu.pl
}

\begin{abstract}
Necessary and sufficient conditions for the positivity and reachability of fractional descriptor positive discrete-time linear systems are established. The minimum energy control problem for descriptor positive systems is formulated and solved. Sufficient conditions for the existence of a solution to the minimum energy control problem are given. A procedure for computation of optimal input sequences and a minimal value of the performance index is proposed and illustrated by a numerical example.
\end{abstract}

Keywords: fractional system, descriptor system, positive system, minimum energy control.

\section{Introduction}

A dynamical system is called positive if its trajectory starting from any nonnegative initial state remains forever in the positive orthant for all nonnegative inputs. Overviews of the state of the art in positive systems theory were made by Farina and Rinaldi (2000) as well as Kaczorek (2001). A variety of models having positive behavior can be found in engineering, economics, social sciences, biology and medicine, etc.

Mathematical fundamentals of fractional calculus are given by Oldham and Spanier (1974), Ostalczyk (2008) as well as Podlubny (1999). Positive fractional linear systems were investigated by Kaczorek (2008a; 2011b; 2011c; 2012). The stability of fractional linear 1D discrete-time and continuous-time systems was investigated by Busłowicz (2008), Dzieliński and Sierociuk (2008) as well as Kaczorek (2012), who also characterized the stability of 2D fractional positive linear systems (Kaczorek, 2009) and introduced the notion of practical stability of positive fractional discrete-time linear systems (Kaczorek, 2008b). Some recent interesting results in fractional systems theory and its applications can be found in the works of Dzieliński et al. (2009), Kaczorek (2011a; 2008c), Radwan et al. (2009), Tenreiro Machado and Ramiro Barbosa (2006) or Vinagre et al. (2002). The minimum energy control problem for standard linear systems was formulated and solved by Klamka $(1991 ; 1983 ; 1976)$ and for 2D linear systems with variable coefficients by Kaczorek and Klamka (1986). The controllability and minimum energy control problems of fractional discrete-time linear systems were investigated by Klamka (2010), while minimum energy control of fractional positive continuous-time linear systems was addressed by Kaczorek (2013a), who also discussed its counterpart for descriptor positive discrete-time linear systems (Kaczorek, 2013b).

In this paper, necessary and sufficient conditions for the positivity and reachability of fractional descriptor systems will be established, and the minimum energy control problem will be formulated and solved.

The paper is organized as follows. In Section 2, the reduction of fractional descriptor linear systems to an equivalent standard system by the use of the shuffle algorithm is addressed. In Section 3, the solution to the standard equivalent fractional system is given, and conditions for the positivity of the fractional descriptor system are established. Necessary and sufficient conditions for the reachability of positive fractional descriptor systems are given in Section 4. The minimum energy control problem is formulated and solved in Section 5. Concluding remarks are given in Section 6.

The following notation will be used: $\mathbb{R}$, the set of real numbers; $\mathbb{R}^{n \times m}$, the set of $n \times m$ real matrices; $\mathbb{R}_{+}^{n \times m}$, the set of $n \times m$ matrices with nonnegative entries and $\mathbb{R}_{+}^{n}=\mathbb{R}_{+}^{n \times 1} ; x^{T}$, the transpose of the vector $x ; I_{n}$, the 
$n \times n$ identity matrix.

\section{Reduction of fractional descriptor systems to standard systems}

Consider the descriptor discrete-time linear system

$$
\begin{aligned}
E \Delta^{\alpha} x_{i+1}= & A x_{i}+B u_{i}, \\
& 0<\alpha<1, \quad i \in \mathbb{Z}_{+}=\{0,1, \ldots\},
\end{aligned}
$$

where

$$
\begin{aligned}
& \Delta^{\alpha} x_{i}=\sum_{j=0}^{i} c_{j} x_{i-j}, c_{j}=(-1)^{j}\left(\begin{array}{l}
\alpha \\
j
\end{array}\right), \\
& \left(\begin{array}{c}
\alpha \\
j
\end{array}\right) \\
& =\left\{\begin{array}{cc}
1 & \text { for } j=0, \\
\frac{\alpha(\alpha-1) \ldots(\alpha-j+1)}{j !} & \text { for } j=1,2, \ldots,
\end{array}\right.
\end{aligned}
$$

is the $\alpha$-th order fractional difference of the state vector, $x_{i} \in \mathbb{R}^{n}$ and $u_{i} \in \mathbb{R}^{m}$ are respectively the state and input vectors, $E, A \in \mathbb{R}^{n \times n}, B \in \mathbb{R}^{n \times m}$.

It is assumed that $\operatorname{det} E=0$ and the pencil $E z-A$ is regular, i.e.,

$$
\operatorname{det}[E z-A] \neq 0 \quad \text { for some } \quad z \in \mathbb{C} \text {. }
$$

Substitution of (2) into (1) yields

$$
\begin{aligned}
E x_{i+1}= & A_{\alpha} x_{i}-E c_{2} x_{i-1}-E c_{3} x_{i-2}-\cdots-E c_{i} x_{1} \\
& -E c_{i+1} x_{0}+B u_{i}, \quad i \in \mathbb{Z}_{+}
\end{aligned}
$$

where $A_{\alpha}=A+E \alpha$.

Theorem 1. If the pencil of the fractional descriptor system (11) is regular (i.e., (3) holds), then the system can be reduced to the standard equivalent form with the use of the shuffle algorithm:

$$
\begin{aligned}
x_{i+1}= & \bar{A}_{i} x_{i}+\bar{A}_{i-1} x_{i-1}+\cdots+\bar{A}_{0} x_{0}+\bar{B}_{0} u_{i} \\
& +\bar{B}_{1} u_{i+1}+\cdots+\bar{B}_{q} u_{i+q},
\end{aligned}
$$

where

$$
\begin{aligned}
& \bar{A}_{k} \in \mathbb{R}_{+}^{n \times n}, \quad k=0,1, \ldots, i, \\
& \bar{B}_{j} \in \mathbb{R}_{+}^{n \times m}, \quad k=0,1, \ldots, q,
\end{aligned}
$$

and $q$ is the number of the shuffles.

Proof. The following elementary row operations will be used (Kaczorek, 1992; 2011):

1. Multiplication of the $i$-th row by a real number $c$. This operation will be denoted by $L[i \times c]$.
2. Addition to the $i$-th row of the $j$-th row multiplied by a real number $c$. This operation will be denoted by $L[i+j \times c]$.

3. Interchange of the $i$-th and $j$-th rows. This operation will be denoted by $L[i, j]$.

Performing elementary row operations on the array

$$
\left[\begin{array}{llllll}
E & A_{\alpha} & -E c_{2} & \ldots & -E c_{i+1} & B
\end{array}\right]
$$

or, equivalently, on (4), we get

$$
\left[\begin{array}{cccccc}
E_{1} & A_{\alpha 1} & -E_{1} c_{2} & \ldots & -E_{1} c_{i+1} & B_{1} \\
0 & A_{\alpha 2} & 0 & \ldots & 0 & B_{2}
\end{array}\right]
$$

and

$$
\begin{aligned}
E_{1} x_{i+1}= & A_{\alpha 1} x_{i}-E_{1} c_{2} x_{i-1}-E_{1} c_{3} x_{i-2} \\
& -\cdots-E_{1} c_{i+1} x_{0}+B_{1} u_{i}, \\
0= & A_{\alpha 2} x_{i}+B_{2} u_{i} .
\end{aligned}
$$

Substituting $i$ in (8b) by $i+1$, we obtain

$$
-A_{\alpha 2} x_{i+1}=B_{2} u_{i+1} \text {. }
$$

Equations (8a) and (9) can be written in the form

$$
\begin{aligned}
{\left[\begin{array}{c}
E_{1} \\
-A_{\alpha 2}
\end{array}\right] x_{i+1}=} & {\left[\begin{array}{c}
A_{\alpha 1} \\
0
\end{array}\right] x_{i}+\left[\begin{array}{c}
-E_{1} c_{2} \\
0
\end{array}\right] x_{i-1} } \\
& +\cdots+\left[\begin{array}{c}
-E_{1} c_{i+1} \\
0
\end{array}\right] x_{0}+\left[\begin{array}{c}
B_{1} \\
0
\end{array}\right] u_{i} \\
& +\left[\begin{array}{c}
0 \\
B_{2}
\end{array}\right] u_{i+1} .
\end{aligned}
$$

The array

$$
\left[\begin{array}{ccccccc}
E_{1} & A_{\alpha 1} & -E_{1} c_{2} & \ldots & -E_{1} c_{i+1} & B_{1} & 0 \\
-A_{\alpha 2} & 0 & 0 & \ldots & 0 & 0 & B_{2}
\end{array}\right]
$$

can be obtained from (7) by performing a shuffle.

If the matrix

$$
\left[\begin{array}{c}
E_{1} \\
-A_{\alpha 2}
\end{array}\right]
$$

is nonsingular, then solving (10) we obtain the standard system

$$
\begin{aligned}
x_{i+1}= & {\left[\begin{array}{c}
E_{1} \\
-A_{\alpha 2}
\end{array}\right]^{-1}\left(\left[\begin{array}{c}
A_{\alpha 1} \\
0
\end{array}\right] x_{i}+\left[\begin{array}{c}
-E_{1} c_{2} \\
0
\end{array}\right] x_{i-1}\right.} \\
& +\cdots+\left[\begin{array}{c}
-E_{1} c_{i+1} \\
0
\end{array}\right] x_{0}+\left[\begin{array}{c}
B_{1} \\
0
\end{array}\right] u_{i} \\
& \left.+\left[\begin{array}{c}
0 \\
B_{2}
\end{array}\right] u_{i+1}\right) .
\end{aligned}
$$


If the matrix is singular, then performing elementary row operations on 11 we obtain

$$
\left[\begin{array}{ccccccc}
E_{2} & A_{\alpha 2} & -E_{2} c_{2} & \ldots & -E_{2} c_{i+1} & B_{2} & B_{4} \\
0 & A_{\alpha 3} & 0 & \ldots & 0 & B_{3} & B_{5}
\end{array}\right]
$$

where $E_{2}$ has full row rank and rank $E_{2} \geq \operatorname{rank} E_{1}$.

Substituting $i$ in $0=A_{\alpha 3} x_{i}+B_{3} u_{i}+B_{5} u_{i+1}$ by $i+1$, we obtain

$$
-A_{\alpha 3} x_{i+1}=B_{3} u_{i+1}+B_{5} u_{i+2} .
$$

The equations

$$
\begin{aligned}
E_{2} x_{i+1}= & A_{\alpha 2} x_{i}-E_{2} c_{2}-\cdots-E_{2} c_{i+1} \\
& +B_{2} u_{i}+B_{4} u_{i+1}
\end{aligned}
$$

and (14) can be written down as

$$
\begin{aligned}
& {\left[\begin{array}{c}
E_{2} \\
-A_{\alpha 3}
\end{array}\right] x_{i+1}} \\
& =\left[\begin{array}{c}
A_{\alpha 2} \\
0
\end{array}\right] x_{i}+\left[\begin{array}{c}
-E_{2} c_{2} \\
0
\end{array}\right] x_{i-1} \\
& \quad+\cdots+\left[\begin{array}{c}
-E_{2} c_{i+1} \\
0
\end{array}\right] x_{0}+\left[\begin{array}{c}
B_{2} \\
0
\end{array}\right] u_{i} \\
& \quad+\left[\begin{array}{c}
B_{4} \\
B_{3}
\end{array}\right] u_{i+1}+\left[\begin{array}{c}
0 \\
B_{5}
\end{array}\right] u_{i+2} .
\end{aligned}
$$

The array

$$
\left[\begin{array}{ccccc}
E_{2} & A_{\alpha 2} & -E_{2} c_{2} & \cdots & \\
-A_{\alpha 3} & 0 & 0 & \cdots & \\
-E_{2} c_{i+1} & B_{2} & B_{4} & 0 \\
0 & 0 & B_{3} & B_{5}
\end{array}\right]
$$

can be obtained from (13) by performing a shuffle.

If

$$
\operatorname{det}\left[\begin{array}{c}
E_{2} \\
-A_{\alpha 3}
\end{array}\right] \neq 0
$$

we can find $x_{i+1}$ from 15 . Otherwise, we repeat the procedure for 16. If the pencil is regular, then after $q$ steps we obtain a nonsingular matrix

$$
\left[\begin{array}{c}
E_{q} \\
-A_{\alpha q+1}
\end{array}\right]
$$

and the desired equation (5).

Example 1. Consider the fractional descriptor system (1) with $\alpha=0.5$ and the matrices

$$
\begin{aligned}
E & =\left[\begin{array}{lll}
1 & 0 & 0 \\
0 & 1 & 0 \\
0 & 0 & 0
\end{array}\right], \\
A & =\left[\begin{array}{ccc}
1 & 0 & 0 \\
0 & 0.5 & 0 \\
0 & 0 & -1
\end{array}\right], \\
B & =\left[\begin{array}{ll}
0 & 1 \\
1 & 0 \\
1 & 1
\end{array}\right] .
\end{aligned}
$$

To reduce the descriptor system to an equivalent standard system using elementary row operations, we reduce the array

$$
\begin{aligned}
& {\left[\begin{array}{cccccccc}
E & A_{\alpha} & -E c_{2} & \ldots & -E c_{i+1} & B
\end{array}\right]} \\
& =\left[\begin{array}{ccccccccc}
1 & 0 & 0 & 1.5 & 0 & 0 & -c_{2} & 0 \\
0 & 1 & 0 & 0 & 1 & 0 & 0 & -c_{2} \\
0 & 0 & 0 & 0 & 0 & -1 & 0 & 0 & \\
0 & \ldots & -c_{i+1} & 0 & 0 & 0 & 1 \\
0 & \ldots & 0 & -c_{i+1} & 0 & 1 & 0 \\
0 & \ldots & 0 & 0 & 0 & 1 & 1
\end{array}\right]
\end{aligned}
$$

to the form (7). In this case, the array (18) has already the desired form (7) with

$$
\begin{aligned}
E_{1} & =\left[\begin{array}{lll}
1 & 0 & 0 \\
0 & 1 & 0
\end{array}\right], \\
A_{\alpha 1} & =\left[\begin{array}{ccc}
1.5 & 0 & 0 \\
0 & 1 & 0
\end{array}\right], \\
E_{1} c_{2} & =\left[\begin{array}{ccc}
c_{2} & 0 & 0 \\
0 & c_{2} & 0
\end{array}\right], \\
& \vdots \\
E_{1} c_{i+1} & =\left[\begin{array}{ccc}
c_{i+1} & 0 & 0 \\
0 & c_{i+1} & 0
\end{array}\right], \\
A_{\alpha 2} & =\left[\begin{array}{lll}
0 & 0 & -1
\end{array}\right], \\
B_{1} & =\left[\begin{array}{cc}
0 & 1 \\
1 & 0
\end{array}\right], \\
B_{2} & =\left[\begin{array}{ll}
1 & 1
\end{array}\right] .
\end{aligned}
$$

Performing the shuffle, we obtain

$$
\begin{aligned}
& {\left[\begin{array}{ccccccc}
E_{1} & A_{\alpha 1} & -E_{1} c_{2} & \ldots & -E_{1} c_{i+1} & B_{1} & 0 \\
-A_{\alpha 2} & 0 & 0 & \ldots & 0 & 0 & B_{2}
\end{array}\right]} \\
& =\left[\begin{array}{cccccccccc}
1 & 0 & 0 & 1.5 & 0 & 0 & -c_{2} & 0 & 0 & \ldots \\
0 & 1 & 0 & 0 & 1 & 0 & 0 & -c_{2} & 0 & \ldots \\
0 & 0 & 1 & 0 & 0 & 0 & 0 & 0 & 0 & \ldots
\end{array}\right. \\
& \left.\begin{array}{ccccccc}
-c_{i+1} & 0 & 0 & 0 & 1 & 0 & 0 \\
0 & -c_{i+1} & 0 & 1 & 0 & 0 & 0 \\
0 & 0 & 0 & 0 & 0 & 1 & 1
\end{array}\right]
\end{aligned}
$$

and the equivalent standard system

$$
\begin{aligned}
x_{i+1}= & \bar{A}_{i} x_{i}+\bar{A}_{i-1} x_{i-1}+\cdots+\bar{A}_{0} x_{0} \\
& +\bar{B}_{0} u_{i}+\bar{B}_{1} u_{i+1}, \quad i \in \mathbb{Z}_{+},
\end{aligned}
$$

where

$$
\bar{A}_{i}=\left[\begin{array}{ccc}
1.5 & 0 & 0 \\
0 & 1 & 0 \\
0 & 0 & 0
\end{array}\right]
$$




$$
\begin{aligned}
\bar{A}_{i-1}= & {\left[\begin{array}{ccc}
-c_{2} & 0 & 0 \\
0 & -c_{2} & 0 \\
0 & 0 & 0
\end{array}\right], } \\
& \vdots \\
\bar{A}_{0}= & {\left[\begin{array}{ccc}
-c_{i+1} & 0 & 0 \\
0 & -c_{i+1} & 0 \\
0 & 0 & 0
\end{array}\right], } \\
\bar{B}_{0}= & {\left[\begin{array}{ll}
0 & 1 \\
1 & 0 \\
0 & 0
\end{array}\right], } \\
\bar{B}_{1}= & {\left[\begin{array}{ll}
0 & 0 \\
0 & 0 \\
1 & 1
\end{array}\right] . }
\end{aligned}
$$

In this case, the number of shuffle is $q=1$.

\section{Solution for the standard equivalent system and the positivity of fractional descriptor systems}

To find a solution $x_{i}$ of the standard discrete-time linear system (5), we shall apply the $\mathcal{Z}$-transform method.

Let $X(z)$ be the $\mathcal{Z}$-transform of $x_{i}$ defined by

$$
\mathcal{Z}\left[x_{i}\right]=\sum_{i=0}^{\infty} x_{i} z^{-i}
$$

Taking into account that

$$
\begin{aligned}
& \mathcal{Z}\left[x_{i-p}\right]=z^{-p} X(z)+z^{-p} \sum_{j=-1}^{-p} x_{j} z^{-j}, \\
& \mathcal{Z}\left[x_{i+p}\right]=z^{p} X(z)-\sum_{l=0}^{p-1} x_{l} z^{p-l},
\end{aligned}
$$

$p=1,2, \ldots$ and applying the $\mathcal{Z}$-transform to Eqn. (5), we obtain

$$
\mathcal{Z}\left[x_{i+1}\right]=\sum_{k=0}^{i} \bar{A}_{k} \mathcal{Z}\left[x_{i-k}\right]+\sum_{j=0}^{q} \bar{B}_{j} \mathcal{Z}\left[u_{i+j}\right]
$$

and

$$
\begin{aligned}
z X(z)-z x_{0}= & \sum_{k=0}^{i} \bar{A}_{k} z^{-k} X(z) \\
& +\sum_{j=0}^{q} \bar{B}_{j} z^{q}\left[U(z)-\sum_{l=0}^{q-1} u_{l} z^{-l}\right]
\end{aligned}
$$

where $U(z)=\mathcal{Z}\left[u_{i}\right]$ and $x_{j}=0, j=-1, \ldots,-k$.
Multiplying (24b) by $z^{-1}$ and solving with respect to $X(z)$, we obtain

$$
\begin{aligned}
X(z)= & {\left[I_{n}-\sum_{k=0}^{i} \bar{A}_{k} z^{-(k+1)}\right]^{-1} } \\
& \times\left[x_{0}+\sum_{j=0}^{q} \bar{B}_{j} z^{q-1}\left[U(z)-\sum_{l=0}^{q-1} u_{l} z^{-l}\right]\right] .
\end{aligned}
$$

Substitution of the expansion

$$
\left[I_{n}-\sum_{k=0}^{i} \bar{A}_{k} z^{-(k+1)}\right]^{-1}=\sum_{j=0}^{\infty} \Phi_{j} z^{-j}
$$

into 25 yields

$$
\begin{aligned}
& X(z) \\
& =\sum_{j=0}^{\infty} \Phi_{j} z^{-j} \\
& \quad \times\left[x_{0}+\sum_{j=0}^{q} \bar{B}_{j} z^{q-1}\left[U(z)-\sum_{l=0}^{q-1} u_{l} z^{-l}\right]\right] .
\end{aligned}
$$

From definition of the inverse matrix, we have

$$
\begin{aligned}
& {\left[I_{n}-\sum_{k=0}^{i} \bar{A}_{k} z^{-(k+1)}\right]\left[\sum_{j=0}^{\infty} \Phi_{j} z^{-j}\right]} \\
& =\left[\sum_{j=0}^{\infty} \Phi_{j} z^{-j}\right]\left[I_{n}-\sum_{k=0}^{i} \bar{A}_{k} z^{-(k+1)}\right]=I_{n} .
\end{aligned}
$$

Comparison of the coefficients at the same powers of $z^{-k}$, $k=0,1, \ldots$ from 28 yields

$$
\begin{array}{ll}
\Phi_{0}=I_{n}, & \Phi_{1}=\bar{A}_{0}, \\
\Phi_{2}=\bar{A}_{0} \Phi_{1}+\bar{A}_{1}, & \Phi_{3}=\bar{A}_{0} \Phi_{2}+\bar{A}_{1} \Phi_{1}+\bar{A}_{2}
\end{array}
$$

and, in general,

$$
\begin{array}{r}
\Phi_{k}=\bar{A}_{0} \Phi_{k-1}+\bar{A}_{1} \Phi_{k-2}+\cdots+\bar{A}_{k-1} \Phi_{0} \\
=\Phi_{k-1} \bar{A}_{0}+\Phi_{k-2} \bar{A}_{1}+\cdots+\Phi_{0} \bar{A}_{k-1}, \\
k=1,2, \ldots
\end{array}
$$

Applying the inverse $\mathcal{Z}$-transform and the convolution theorem (Kaczorek, 2012) to 27), we obtain the desired solution

$$
\begin{aligned}
x_{i}= & \Phi_{i} x_{0} \\
& +\sum_{k=0}^{i} \Phi_{i-k-1}\left(\sum_{j=0}^{q} \bar{B}_{j} u_{j+k}\right), \quad i \in \mathbb{Z}_{+} .
\end{aligned}
$$

Thus, the following theorem has been proved. 
Theorem 2. The solution of Eqn. (5) has the form (30), where the matrices $\Phi_{k}$ are given by (29).

Definition 1. The fractional discrete system (1) is called (internally) positive if $x_{i} \in \mathbb{R}_{+}^{n}$ for every consistent $x_{0} \in$ $\mathbb{R}_{+}^{n}$ and all inputs $u_{i} \in \mathbb{R}_{+}^{m}, i \in \mathbb{Z}_{+}$.

Theorem 3. The fractional descriptor system (1) is positive if and only if the matrices of the equivalent standard system (5) satisfy the conditions

$$
\bar{A}_{k} \in \mathbb{R}_{+}^{n \times n}, \quad k=0,1, \ldots, i
$$

and

$$
\bar{B}_{j} \in \mathbb{R}_{+}^{n \times m}, \quad j=0,1, \ldots, q .
$$

Proof. It is well known that the state vectors of the descriptor system (1) and the equivalent standard system (5) are the same. By Definition 1, the descriptor system (1) is positive if and only if the standard system (5) is positive. From (30) it follows that $x_{i} \in \mathbb{R}_{+}^{n}, i \in \mathbb{Z}_{+}$ if the conditions (31) are met and $x_{0} \in \mathbb{R}_{+}^{n}, u_{i} \in \mathbb{R}_{+}^{m}$. The necessity of the condition (31) follows from the fact that $x_{i} \in \mathbb{R}_{+}^{n}$ for every consistent $x_{0} \in \mathbb{R}_{+}^{n}$ and arbitrary $u_{i} \in \mathbb{R}_{+}^{m}, i \in \mathbb{Z}_{+}$(Kaczorek, 2012).

Example 2. (Continuation of Example 1) Check the positivity of the fractional descriptor system (1) with $\alpha=$ 0.5 and the matrices (17). In Example 1 it was shown that the equivalent standard system has the matrices given by (21a) and (21b). The matrices have nonnegative entries since

$$
c_{2}=\frac{\alpha(\alpha-1)}{2 !}<0 \quad \text { for } \quad \alpha=0.5
$$

and

$$
\begin{aligned}
c_{k+1} & =(-1)^{k+1} \frac{\alpha(\alpha-1) \ldots(\alpha-k+1)(\alpha-k)}{(k+1) !} \\
& =c_{k} \frac{k-\alpha}{k+1}<0, \quad k=2,3, \ldots
\end{aligned}
$$

Therefore, the fractional descriptor system (1) with $\alpha=0.5$ and 17 is positive.

\section{Reachability of positive fractional descriptor systems}

Consider the positive fractional descriptor discrete-time system (1). It is called reachable in $n$ steps if and only if the equivalent standard system (5) is reachable in $n$ steps.

Definition 2. The positive system (5) is called reachable in $n$ steps if for any given $x_{f} \in \mathbb{R}_{+}^{n}$ there exists an input sequence $u_{k} \in \mathbb{R}_{+}^{m}$ for $k=0,1, \ldots, h-1, h=n+q$, which steers the state of the system from $x_{0}=0$ to $x_{f} \in$ $\mathbb{R}_{+}^{n}$, i.e., $x_{n}=x_{f}$.
Theorem 4. The positive system (5) is reachable in $n$ steps if and only if the reachability matrix

$$
\begin{aligned}
R_{n}= & {\left[\begin{array}{ccc}
\Phi_{n-1} \bar{B}_{0} & \Phi_{n-2} \bar{B}_{1} & \ldots \\
& \Phi_{n-q-2} \bar{B}_{q-1} & \Phi_{n-q-1} \bar{B}_{q}
\end{array}\right] }
\end{aligned}
$$

contains $n$ linearly independent monomial columns.

Proof. Using (30) for $i=n$ and $x_{0}=0$, we obtain

$$
\begin{aligned}
& x_{f}=x_{n} \\
& =\left[\begin{array}{lll}
\Phi_{n-q-1} \bar{B}_{q} & \Phi_{n-q-2} \bar{B}_{q-1} & \ldots
\end{array}\right. \\
& \left.\Phi_{n-2} \bar{B}_{1} \quad \Phi_{n-1} \bar{B}_{0}\right]\left[\begin{array}{c}
u_{n+q} \\
u_{n+q-1} \\
\vdots \\
u_{0}
\end{array}\right] \\
& =R_{n}\left[\begin{array}{c}
u_{0} \\
u_{1} \\
\vdots \\
u_{h+q}
\end{array}\right] \text {, }
\end{aligned}
$$

where $R_{n}$ is defined by (33).

From (34) it follows that there exists an input sequence $u_{k} \in \mathbb{R}_{+}^{m}$ for $k=0,1, \ldots, i+q$ if and only if the matrix (33) contains $n$ linearly independent monomial columns.

Remark 1. Assuming that the components of the input sequence which do not correspond to the chosen linear independent monomial columns are zero, we obtain a different input sequence which steers the state vector from $x_{0}=0$ to $x_{n}=x_{f}$.

Example 3. (Continuation of Examples 1 and 2) Check the reachability of the positive fractional descriptor system (1) with $\alpha=0.5$ and the matrices (17), and compute an input sequence that steers in two steps the state vector of the system from $x_{0}=\left[\begin{array}{lll}0 & 0 & 0\end{array}\right]^{T}$ to $x_{f}=\left[\begin{array}{lll}1 & 1 & 1\end{array}\right]^{T}$. In this case, the reachability matrix (33) has the form

$$
\begin{aligned}
R_{4} & =\left[\begin{array}{ccccc}
\bar{B}_{1} & \bar{B}_{0}+\bar{A}_{1} \bar{B}_{1} & \bar{A}_{1} \bar{B}_{0}
\end{array}\right] \\
& =\left[\begin{array}{cccccc}
0 & 0 & 0 & 1 & 0 & 1.5 \\
0 & 0 & 1 & 0 & 1 & 0 \\
1 & 1 & 0 & 0 & 0 & 0
\end{array}\right] .
\end{aligned}
$$

The matrix (35) contains three linearly independent monomial columns, and by Theorem 4 the positive fractional descriptor system is reachable in two steps. Choosing as linearly independent monomial columns the fifth, fourth and first column of the matrix (35), we obtain

$$
\left[\begin{array}{lll}
0 & 1 & 0 \\
0 & 0 & 1 \\
1 & 0 & 0
\end{array}\right]\left[\begin{array}{l}
u_{21} \\
u_{12} \\
u_{01}
\end{array}\right]=\left[\begin{array}{l}
1 \\
1 \\
1
\end{array}\right],
$$




$$
\left[\begin{array}{l}
u_{21} \\
u_{12} \\
u_{01}
\end{array}\right]=\left[\begin{array}{lll}
0 & 1 & 0 \\
0 & 0 & 1 \\
1 & 0 & 0
\end{array}\right]^{-1}\left[\begin{array}{l}
1 \\
1 \\
1
\end{array}\right]=\left[\begin{array}{l}
1 \\
1 \\
1
\end{array}\right]
$$

and

$$
\left[\begin{array}{l}
u_{2} \\
u_{1} \\
u_{0}
\end{array}\right]=\left[\begin{array}{l}
u_{21} \\
u_{22} \\
u_{11} \\
u_{12} \\
u_{01} \\
u_{02}
\end{array}\right]=\left[\begin{array}{l}
1 \\
0 \\
0 \\
1 \\
1 \\
0
\end{array}\right]
$$

If we choose as linearly independent monomial columns the second, third and last ones of the matrix (35), then we obtain

$$
\left[\begin{array}{l}
u_{21} \\
u_{12} \\
u_{01}
\end{array}\right]=\left[\begin{array}{ccc}
0 & 0 & 1.5 \\
0 & 1 & 0 \\
1 & 0 & 0
\end{array}\right]^{-1}\left[\begin{array}{l}
1 \\
1 \\
1
\end{array}\right]=\left[\begin{array}{c}
1 \\
1 \\
\frac{10}{15}
\end{array}\right]
$$

and

$$
\left[\begin{array}{l}
u_{2} \\
u_{1} \\
u_{0}
\end{array}\right]=\left[\begin{array}{l}
u_{21} \\
u_{22} \\
u_{11} \\
u_{12} \\
u_{01} \\
u_{02}
\end{array}\right]=\left[\begin{array}{c}
1 \\
0 \\
0 \\
1 \\
0 \\
\frac{10}{15}
\end{array}\right]
$$

\section{Minimum energy control problem}

Consider the fractional descriptor positive system (1) reduced to the form (2). In Section 4 it was shown that if the positive system is reachable then there exist many input sequences which steer the state of the system from $x_{0}=0$ to the given final state $x_{f} \in \mathbb{R}_{+}^{n}$. Among these input sequences we are looking for a sequence $u_{k} \in \mathbb{R}_{+}^{m}$ for $k=0,1, \ldots, n+q-1$ that minimizes the performance index

$$
I(u)=\sum_{i=0}^{h-1} u_{i}^{T} Q u_{i}
$$

where $Q \in \mathbb{R}_{+}^{m \times m}$ is a symmetric positive definite matrix such that

$$
Q^{-1} \in \mathbb{R}_{+}^{m \times m},
$$

and $h=n+q$ is the number of steps in which the state of the system is transferred from $x_{0}=0$ to the given final state $x_{f} \in \mathbb{R}_{+}^{n}$.

The minimum energy control problem for the fractional descriptor positive discrete-time linear system (1) can be stated as follows.

Given the matrices $E, A, B$ of the descriptor positive system (1), the number of steps $h$, the final state $x_{f} \in \mathbb{R}_{+}^{n}$ and the matrix $Q \in \mathbb{R}_{+}^{n \times n}$ of the performance index (38) satisfying the condition (39), find a sequence of inputs $u_{k} \in \mathbb{R}_{+}^{m}$ for $k=0,1, \ldots, h-1$ that steers the state of the system from $x_{0}=0$ to $x_{f} \in \mathbb{R}_{+}^{n}$ and minimizes the performance index (38).

To solve the problem, we define the matrix

$$
W_{h}=R_{h} Q_{h}^{-1} R_{h}^{T} \in \mathbb{R}_{+}^{n \times n},
$$

where $R_{h} \in \mathbb{R}_{+}^{n \times h m}$ is given by (33) and

$$
Q_{h}^{-1}=\text { block } \operatorname{diag}\left[Q^{-1}, \ldots, Q^{-1}\right] \in \mathbb{R}_{+}^{h m \times h m} .
$$

The matrix (40) is non-singular if the positive system is reachable in $h$ steps.

For a given $x_{f} \in \mathbb{R}_{+}^{n}$, we may define the input sequence

$$
\hat{u}=\left[\begin{array}{c}
\hat{u}_{h-1} \\
\hat{u}_{h-2} \\
\vdots \\
\hat{u}_{0}
\end{array}\right]=Q_{h}^{-1} R_{h}^{T} W_{h}^{-1} x_{f}
$$

where $Q_{h}^{-1}, W_{h}$ and $R_{h}$ are defined by (41), (40) and (33), respectively.

Note that $\hat{u} \in \mathbb{R}_{+}^{h m}$ if

$$
W_{h}^{-1} \in \mathbb{R}_{+}^{n \times n},
$$

and this holds if the condition (39) is met.

Theorem 5. Let the fractional descriptor positive system (11) be reachable in $h$ steps and the conditions (39) and (43) be satisfied. Moreover, let

$$
\bar{u}=\left[\begin{array}{c}
\bar{u}_{h-1} \\
\bar{u}_{h-2} \\
\vdots \\
\bar{u}_{0}
\end{array}\right] \in \mathbb{R}_{+}^{h m},
$$

be an input sequence that steers the state of the system from $x_{0}=0$ to $x_{f} \in \mathbb{R}_{+}^{n}$. Then the input sequence (42) also steers the state of the system from $x_{0}=0$ to $x_{f} \in \mathbb{R}_{+}^{n}$ and minimizes the performance index (38), i.e.,

$$
I(\hat{u}) \leq I(\bar{u})
$$

The minimal value of the performance index (38) is given by

$$
I(\hat{u})=x_{f}^{T} W_{h}^{-1} x_{f}
$$

Proof. If the conditions (39) and 43 are met and the system is reachable in $h$ steps, then the input sequence (42) is well defined and $\hat{u} \in \mathbb{R}_{+}^{n}$. We shall show that the input sequence (42) steers the state of the system from $x_{0}=0$ to $x_{f} \in \mathbb{R}_{+}^{n}$. Using (35) and (42), we obtain

$$
x_{h}=R_{h} \hat{u}=R_{h} Q_{h}^{-1} R_{h}^{T} W_{h}^{-1} x_{f}=x_{f},
$$


since, by (40), $R_{h} Q_{h}^{-1} R_{h}^{T}=W_{h}$. Hence, $x_{f}=R_{h} \hat{u}=$ $R_{h} \bar{u}$ or

$$
R_{h}[\hat{u}-\bar{u}]=0 .
$$

The transposition of (48) yields

$$
[\hat{u}-\bar{u}]^{T} R_{h}^{T}=0
$$
obtain

Postmultiplying the equality 496 by $W_{h}^{-1} x_{f}$, we

$$
[\hat{u}-\bar{u}]^{T} R_{h}^{T} W_{h}^{-1} x_{f}=0 .
$$

From (42), we have $Q_{h} \hat{u}=R_{h}^{T} W_{h}^{-1} x_{f}$. Substitution of this equality into (50) yields

$$
[\hat{u}-\bar{u}]^{T} Q_{h} \hat{u}=0
$$

where $Q_{h}=$ block diag $[Q, \ldots, Q] \in \mathbb{R}_{+}^{h m \times h m}$.

From (51), it follows that

$$
\bar{u}^{T} Q_{h} \bar{u}=\hat{u}^{T} Q_{h} \hat{u}+[\bar{u}-\hat{u}]^{T} Q_{h}[\bar{u}-\hat{u}],
$$

since, by (51), $\bar{u}^{T} Q_{h} \hat{u}=\hat{u}^{T} Q_{h} \hat{u}=\hat{u}^{T} Q_{h} \bar{u}$.

From (52) it follows that (45) holds since $[\bar{u}-$ $\hat{u}]^{T} Q_{h}[\bar{u}-\hat{u}] \geq 0$. To find the minimal value of the performance index (38), we substitute (42) into (38) and obtain

$$
\begin{aligned}
I(\hat{u}) & =\sum_{i=0}^{h-1} \hat{u}_{i}^{T} Q \hat{u}_{i}=\hat{u}^{T} Q_{h} \hat{u} \\
& =\left[Q_{h}^{-1} R_{h}^{T} W_{h}^{-1} x_{f}\right]^{T} Q_{h}\left[Q_{h}^{-1} R_{h}^{T} W_{h}^{-1} x_{f}\right] \\
& =x_{f}^{T} W_{h}^{-1} R_{h} Q_{h}^{-1} R_{h}^{T} W_{h}^{-1} x_{f} \\
& =x_{f}^{T} W_{h}^{-1} x_{f}
\end{aligned}
$$

since, by, (40) $W_{h}^{-1} R_{h} Q_{h}^{-1} R_{h}^{T}=I_{n}$.

From the above discussion we have the following procedure for computation of the optimal input sequence and the minimal value of the performance index (42).

\section{Procedure 1.}

Step 1. Knowing $E, A, B$, compute $\bar{A}_{k}$ for $k=0,1, \ldots, i$ and $\bar{B}_{l}$ for $l=0,1, \ldots, q$.

Step 2. Using (33), compute $R_{h}$.

Step 3. For given $Q$, using (41), compute $Q_{h}^{-1}$.

Step 4. Using (40), compute $W_{h}$ and $W_{h}^{-1}$.

Step 5. For given $x_{f} \in \mathbb{R}_{+}^{n}$, using (42), compute $\hat{u}$.

Step 6. Using (46), compute the minimal value of the performance index $I(\hat{u})$.

Example 4. (Continuation of Examples 1-3) For the fractional descriptor system (1) with $\alpha=0.5$ and (17), compute the optimal input sequence that steers the system from $x_{0}=\left[\begin{array}{lll}0 & 0 & 0\end{array}\right]^{T}$ to $x_{f}=\left[\begin{array}{lll}1 & 1 & 1\end{array}\right]^{T}$, and minimize the performance index (38) for

$$
Q_{h}=\operatorname{diag}[2,2,2,2] .
$$

Using Procedure 1 and results of Example 3, we obtain the following.

Step 1. The desired matrices $\bar{A}_{k}$ for $k=0,1, \ldots, i$ and $\bar{B}_{l}$ for $l=0,1$ are given by (21).

Step 2. The matrix $R_{4}$ is given by (35).

Step 3. Using (41) and (54), we obtain

$$
Q_{4}^{-1}=\operatorname{diag}[0.5,0.5,0.5,0.5,0.5,0.5] \text {. }
$$

Step 4. Using (40) and (55), we obtain

$$
\begin{aligned}
W_{4}= & R_{4} Q_{4}^{-1} R_{4}^{T} \\
= & {\left[\begin{array}{cccccc}
0 & 0 & 0 & 1 & 0 & 1.5 \\
0 & 0 & 1 & 0 & 1 & 0 \\
1 & 1 & 0 & 0 & 0 & 0
\end{array}\right] } \\
& {\left[\begin{array}{cccccc}
0.5 & 0 & 0 & 0 & 0 & 0 \\
0 & 0.5 & 0 & 0 & 0 & 0 \\
0 & 0 & 0.5 & 0 & 0 & 0 \\
0 & 0 & 0 & 0.5 & 0 & 0 \\
0 & 0 & 0 & 0 & 0.5 & 0 \\
0 & 0 & 0 & 0 & 0 & 0.5
\end{array}\right] } \\
& \times\left[\begin{array}{ccc}
0 & 0 & 1 \\
0 & 0 & 1 \\
0 & 1 & 0 \\
1 & 0 & 0 \\
0 & 1 & 0 \\
1.5 & 0 & 0
\end{array}\right]=\left[\begin{array}{cccc}
1.625 & 0 & 0 \\
0 & 1 & 0 \\
0 & 0 & 1
\end{array}\right]
\end{aligned}
$$

and

$$
W_{4}^{-1}=\left[\begin{array}{ccc}
0.6154 & 0 & 0 \\
0 & 1 & 0 \\
0 & 0 & 1
\end{array}\right] \text {. }
$$

Step 5. Using (42), 55) and (56b), we obtain

$$
\begin{aligned}
\hat{u} & =\left[\begin{array}{c}
\hat{u}_{2} \\
\hat{u}_{1} \\
\hat{u}_{0}
\end{array}\right]=Q_{4}^{-1} R_{4}^{T} W_{4}^{-1} x_{f} \\
& =\left[\begin{array}{cccccc}
0.5 & 0 & 0 & 0 & 0 & 0 \\
0 & 0.5 & 0 & 0 & 0 & 0 \\
0 & 0 & 0.5 & 0 & 0 & 0 \\
0 & 0 & 0 & 0.5 & 0 & 0 \\
0 & 0 & 0 & 0 & 0.5 & 0 \\
0 & 0 & 0 & 0 & 0 & 0.5
\end{array}\right]
\end{aligned}
$$




$$
\begin{aligned}
& =\left[\begin{array}{lll}
0 & 0 & 1 \\
0 & 0 & 1 \\
0 & 1 & 0 \\
1 & 0 & 0 \\
0 & 1 & 0 \\
1.5 & 0 & 0
\end{array}\right]\left[\begin{array}{ccc}
0.6154 & 0 & 0 \\
0 & 1 & 0 \\
0 & 0 & 1
\end{array}\right]\left[\begin{array}{l}
1 \\
1 \\
1
\end{array}\right] \\
& =\left[\begin{array}{c}
0.5 \\
0.4615 \\
0.5 \\
0.3077 \\
0.5 \\
0.5
\end{array}\right] .
\end{aligned}
$$

Step 6. Using (46) and (56b), we obtain

$$
\begin{aligned}
I(\hat{u}) & =x_{f}^{T} W_{4}^{-1} x_{f} \\
& =\left[\begin{array}{lll}
1 & 1 & 1
\end{array}\right]\left[\begin{array}{ccc}
0.6154 & 0 & 0 \\
0 & 1 & 0 \\
0 & 0 & 1
\end{array}\right]\left[\begin{array}{l}
1 \\
1 \\
1
\end{array}\right] \\
& =2.6154 .
\end{aligned}
$$

Note that the optimal input sequence (58) is different from the input sequences (36) and (37).

\section{Concluding remarks}

Necessary and sufficient conditions for the positivity and reachability of fractional descriptor positive discrete-time linear systems have been established (Theorems 1 and Theorem 3). The transformation of the fractional descriptor system to an equivalent standard one with the use of the shuffle algorithm has been addressed. The minimum energy control problem for fractional descriptor positive systems has been formulated and solved (Theorem 5). A procedure for computation of optimal input sequences and a minimal value of the performance index has been proposed (Procedure 1). The procedure has been demonstrated on a numerical example.

An open problem is the extension of these deliberations to fractional positive descriptor 2D continuous-discrete linear systems.

\section{Acknowledgment}

This work was supported by the National Science Centre in Poland under the work G/WE/1/11.

\section{References}

Busłowicz, M. (2008). Stability of linear continuous time fractional order systems with delays of the retarded type, Bulletin of the Polish Academy of Sciences: Technical Sciences 56(4): 319-324.

Dzieliński, A. and Sierociuk, D. (2008). Stability of discrete fractional order state-space systems, Journal of Vibrations and Control 14(9/10): 1543-1556.
Dzieliński, A., Sierociuk, D. and Sarwas, G. (2009). Ultracapacitor parameters identification based on fractional order model, Proceedings of ECC'09, $\mathrm{Bu}$ dapest, Hungary, pp. 196-200.

Farina, L. and Rinaldi, S. (2000). Positive Linear Systems: Theory and Applications, J. Wiley, New York, NY.

Kaczorek, T. (1992). Linear Control Systems, Research Studies Press/J. Wiley, New York, NY.

Kaczorek, T. (2001). Positive $1 D$ and 2D Systems, Springer-Verlag, London.

Kaczorek, T. (2008a). Fractional positive continuous-time systems and their reachability, International Journal of Applied Mathematics and Computer Science 18(2): 223-228, DOI: $10.2478 / \mathrm{v} 10006-008-0020-0$

Kaczorek, T. (2008b). Practical stability of positive fractional discrete-time linear systems, Bulletin of the Polish Academy of Sciences: Technical Sciences 56(4): 313-318.

Kaczorek, T. (2008c). Reachability and controllability to zero tests for standard and positive fractional discrete-time systems, Journal Européen des Systèmes Automatisés 42(6-8): 769-787.

Kaczorek, T. (2009). Asymptotic stability of positive fractional 2D linear systems, Bulletin of the Polish Academy of Sciences: Technical Sciences 57(3): 289-292.

Kaczorek, T. (2011a). Controllability and observability of linear electrical circuits, Electrical Review 87(9a): 248-254.

Kaczorek, T. (2011b). Positivity and reachability of fractional electrical circuits, Acta Mechanica et Automatica 5(2): $42-51$.

Kaczorek, T. (2011c). Positive linear systems consisting of $n$ subsystems with different fractional orders, IEEE Transactions on Circuits and Systems 58(6): 1203-1210.

Kaczorek, T. (2011d). Checking of the positivity of descriptor linear systems by the use of the shuffle algorithm, Archives of Control Sciences 21(3): 287-298.

Kaczorek, T. (2012). Selected Problems of Fractional Systems Theory, Springer-Verlag, Berlin.

Kaczorek, T. (2013a). Minimum energy control of fractional positive continuous-time linear systems, MMAR Conference, Międzyzdroje, Poland, pp. 622-626.

Kaczorek, T. (2013b). Minimum energy control of descriptor positive discrete-time linear systems, COMPEL 33(2): $1-14$.

Kaczorek, T. and Klamka, J. (1986). Minimum energy control of 2D linear systems with variable coefficients, International Journal of Control 44(3): 645-650.

Klamka J., (1976). Relative controllability and minimum energy control of linear systems with distributed delays in control, IEEE Transactions on Automatic Control 21(4): 594-595.

Klamka, J. (1983). Minimum energy control of 2D systems in Hilbert spaces, System Sciences 9(1-2): 33-42.

Klamka, J. (1991). Controllability of Dynamical Systems, Kluwer Academic Press, Dordrecht. 
Klamka, J. (2010). Controllability and minimum energy control problem of fractional discrete-time systems, in D. Baleanu, Z.B. Guvenc and J.A. Tenreiro Machado (Eds.), New Trends in Nanotechnology and Fractional Calculus, Springer-Verlag, New York, NY, pp. 503-509.

Oldham, K.B. and Spanier, J. (1974). The Fractional Calculus, Academic Press, New York, NY.

Ostalczyk, P. (2008). Epitome of the Fractional Calculus: Theory and Its Applications in Automatics, Technical University of Łódź Press, Łódź, (in Polish).

Podlubny, I. (1999). Fractional Differential Equations, Academic Press, San Diego, CA.

Radwan, A.G., Soliman, A.M., Elwakil, A.S. and Sedeek, A. (2009). On the stability of linear systems with fractional-order elements, Chaos, Solitons and Fractals 40(5): 2317-2328.

Tenreiro Machado J.A., Ramiro Barbosa S., (2006). Functional dynamics in genetic algorithms, Workshop on Fractional Differentiation and Its Application, Porto, Portugal, Vol. 1, pp. 439-444.

Vinagre B.M., Monje C.A., Calderon A.J. (2002). Fractional order systems and fractional order control actions, IEEE CDC'02, Las Vegas, NV, USA, TW\#2, Lecture 3.

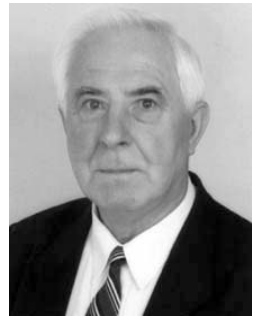

Tadeusz Kaczorek received the M.Sc., Ph.D. and D.Sc. degrees in electrical engineering from the Warsaw University of Technology in 1956, 1962 and 1964, respectively. In the years 1968-69 he was the dean of the Electrical Engineering Faculty, and in the period of 1970-73 he was a deputy rector of the Warsaw University of Technology. In 1971 he became a professor and in 1974 a full professor at the same university. Since 2003 he has been a professor at the Białystok University of Technology. In 1986 he was elected a corresponding member and in 1996 a full member of the Polish Academy of Sciences. In the years 1988-1991 he was the director of the Research Centre of the Polish Academy of Sciences in Rome. In 2004 he was elected an honorary member of the Hungarian Academy of Sciences. He has been granted honorary doctorates by 11 universities. His research interests cover systems theory, especially singular multidimensional systems, positive multidimensional systems, singular positive 1D and 2D systems, as well as positive fractional 1D and 2D systems. He initiated research in the field of singular 2D, positive 2D and positive fractional linear systems. He has published 28 books ( 8 in English) and over 1000 scientific papers. He has also supervised $69 \mathrm{Ph} . \mathrm{D}$. theses. He is the editor-in-chief of the Bulletin of the Polish Academy of Sciences: Technical Sciences and a member of editorial boards of ten international journals.

Received: 21 November 2013 Revised: 22 January 2014 